\title{
Regime identification in ASDEX Upgrade
}

\author{
L.Giannone, A.C.C.Sips, O.Kardaun, G.Pautasso, F.Spreitler, W.Suttrop, \\ C.Tichmann and the ASDEX Upgrade Team \\ Max Planck Institut für Plasmaphysik, \\ EURATOM-IPP Association, D-85748 Garching, Germany
}

\section{Introduction}

The ability to recognise the transition from one state to another reliably from a conveniently small number of measurements in real time is of increasing importance for machine safety and performance control. Previous studies have been carried out using data from TCV [1] and the ITER H-mode database [2]. This work is based on methods of discriminant analysis developed previously [3] and extends investigations at ASDEX Upgrade [4].

Discriminant analysis is concerned with the problem of determining a rule, from a data set of observations which have been classified into distinct groups, that allows the group membership of a subsequent observation to be decided. One example, from speech recognition, was concerned with several repeated utterances of a word by a group of people, from which acoustic parameters are allocated. This word then had to be identified from a speaker not in the group used for training [5]. Discriminant analysis has been applied to regime identification of plasma discharges in the ASDEX Upgrade tokamak. An observation consists of a set of plasma parameters averaged over a time slice in a discharge. The data set consists of all observations over different discharges and time slices and the assignment of this time slice to a particular regime. The aim is to predict the class or regime of a new observation from the measured plasma parameters. The coefficient matrix produced by regime identification provides an efficient means of deciding if a set of plasma parameters is in a particular regime. It is more efficient than applying a scaling law to distinguish between the L-mode and $\mathrm{H}$-mode and can be applied to cases where no scaling law exists.

\section{L-mode and H-mode}

Two energy confinement regimes were identified on the ASDEX tokamak [6]. Above a power threshold of the injected neutral beam power, the energy confinement time increased. This regime with the lower energy confinement time (L-mode) and the higher energy confinement time (H-mode) can also be observed on present large tokamaks such as ASDEX Upgrade and JET. A further increase in the energy confinement time is possible by tailoring the magnetic shear in the plasma centre (improved H-mode) [7]. Discriminant analysis is applied to recognise the transition from the $\mathrm{L}$-mode to the $\mathrm{H}$-mode and from the $\mathrm{H}$-mode to the improved H-mode. This would be valuable for real time machine control, as it would allow appropriate action to be taken to keep a discharge in the desired energy confinement regime.

A data set of the average of plasma parameters over time slices of ASDEX Upgrade discharges has been collected and the confinement regime for each time slice was allocated. Data from 3017 time slices, with 2383 allocated to H-mode and 634 to L-mode, were collected in the AUG Topical Database. Discriminant analysis yields coefficients allowing the 
classification of a new observation. An automated search was performed over 21 plasma variables to find the linear combination of plasma variables that minimises the failure rate of prediction of the assigned regime of the time slices. The linear combination of 5 plasma parameters $\left(\mathrm{U}_{\text {loop }}, \mathrm{q}_{95}, \mathrm{P}_{L}, \mathrm{I}_{p}, \beta_{p}\right.$ ) yielded a minimum prediction failure rate of $1.3 \%$ of those time slices in the training set.

Discriminant analysis allows the probabilities to be calculated for the L-mode and $\mathrm{H}$ mode, as shown on an expanded time scale in Figure 1. The prediction for the H-mode is about $15 \mathrm{~ms}$ after the actual $\mathrm{H}$-mode transition indicated by the dashed line at $\mathrm{t}=1.62 \mathrm{~s}$. In this discharge, the $20 \mathrm{~ms}$ time slices in the training set are allocated to the L-mode at $\mathrm{t}=1.37 \mathrm{~s}$ and $\mathrm{t}=1.47 \mathrm{~s}$ and to the H-mode at $\mathrm{t}=2.7 \mathrm{~s}, \mathrm{t}=3.9 \mathrm{~s}$ and $\mathrm{t}=4.9 \mathrm{~s}$. It is not to be expected that the predicted transition time to the H-mode be exactly at the experimentally observed transition time given that this analysis should produce a best fit of the transitions in the training set. The consequences of this time difference for real time control will be a subject of interest when discrimination analysis is actually implemented on ASDEX Upgrade.

\section{H-mode and improved H-mode}

The improved H-mode in ASDEX Upgrade is one of the advanced tokamak scenarios being developed for stationary high performance operation [7]. A q profile with low magnetic shear and central value, $\mathrm{q}(0)$, close to 1 is the key to obtaining the improved H-mode. A 2.5 MW NBI source is switched on during the current ramp up at low density $\left(\bar{n}_{e}<\right.$ $3 \times 10^{19} \mathrm{~m}^{-3}$ ) to increase the current diffusion time and delay the formation of an $\mathrm{m}=1$ and $\mathrm{n}=1$ resonant surface. Once in a lower single null configuration, sawteeth are inhibited by NBI driven MHD fishbone activity.

The data set contained 605 observations, with 329 improved H-mode and 276 ordinary H-mode time slices. From a random subset of these observations, a training set was chosen and discriminant analysis was performed to find the linear combination of plasma variables that minimised the prediction failure rate of the allocated confinement regime. The linear combination of 5 plasma parameters $\left(\mathrm{P}_{L}, \mathrm{~B}_{T}, \mathrm{a}, \beta_{N}, \beta_{p}\right)$ yielded a minimum prediction failure rate of $5.3 \%$ of those time slices in the training set.

The probabilities calculated for the improved H-mode and ordinary H-mode are shown on an expanded time scale in Figure 2. Between $2.1 \mathrm{~s}$ and $2.2 \mathrm{~s}$ is the time where the density and temperature profiles evolve as a result of the transition to the improved $\mathrm{H}$-mode. The time slices from this discharge in the training set are at $1.7 \mathrm{~s}$ for the ordinary $\mathrm{H}$-mode and $2.5 \mathrm{~s}$ for the improved $\mathrm{H}$-mode. In this case, the transition time of the $\mathrm{H}$-improved probability trace falls within the time window where the plasma is evolving towards an equilibrium state.

\section{Disruptions}

Regime identification was applied to disruption prediction in tokamak discharges. A reliable warning of an imminent disruption is desirable for machine safety. From 61 discharges, time slices were chosen to represent the safe regime and the disruption regime of the discharge. The matrix coefficients derived by discriminant analysis were subsequently applied to these discharges to illustrate the use of regime identification for disruption prediction. In Figure 3, an overview of the time evolution of a disruptive discharge is shown. The time slices from this discharge in the training set are at $2.74 \mathrm{~s}$ for the safe regime and $3.30 \mathrm{~s}$ for the disruption regime. The linear combination of 5 plasma parameters $\left(\mathrm{U}_{\text {loop }}, \beta_{N}, \beta_{p}, l_{i}\right.$, $\mathrm{dW}_{M H D} / \mathrm{dt}$ ) yielded a minimum prediction failure rate of $3.8 \%$ of those time slices in the training set. In the bottom most plot, the regime probabilities for the safe regime (green) and 
disruption regime (red) are shown. In Figure 4, the time evolution of plasma parameters close to the time of disruption are shown on an expanded time scale. A review of the 61 discharges has shown that the disruption alarm should be issued when the disruption probability reaches 0.93. Otherwise, minor disruptions would sometimes produce a false alarm. In addition, prior to allowing the disruption warning to be issued, it is necessary to satisfy the condition that the disruption probability remains below 0.2 for $0.1 \mathrm{~s}$ or below 0.5 for $0.2 \mathrm{~s}$, to prevent false alarms at the start of the discharge.

\section{Discussion}

Although more variables were available, discrimination analysis could be successfully performed with 5 plasma parameters. These plasma parameters vary with the regime transition considered and are those parameters relevant to the physics of the transition. The prediction failure rate decreased with an increasing number of plasma parameters. Above 5 plasma parameters, the decrease in prediction failure rate was slower. Using 5 plasma parameters for discrimination analysis is a compromise between reducing the prediction failure rate and minimising the number of matrix coefficients in the probability calculation $\left(\frac{1}{2} n(n-1)\right)$ so that the number of operations for the real time control system is minimised.

When performing discriminant analysis on a random $60 \%$ sample of the data set, it was found that the prediction failure rate on the remaining $40 \%$ of the data set or on the whole data set were to within error bars the same. The small difference in prediction failure rate when taking the full data set or a random $60 \%$ sample of the data set shows that the regime identification procedure is robust when applied to data not in the training set.

The typicality is a statistical value to quantify how well a new observation belongs to either regime of the training set. This value was used to reject a new observation in discharges with plasma parameters considered not representative of either regime in the the training set. It was also useful in identifying those time slices with an incorrectly allocated regime.

Neural networks have also been applied to disruption prediction in tokamaks $[8,9]$ and work is in progress to compare both methods. Using neural networks, $85 \%$ of the disruptions were successfully predicted by correctly issuing a warning more than $50 \mathrm{~ms}$ before the disruption. Using regime identification, $89 \%$ of the warnings were correctly issued more than $50 \mathrm{~ms}$ before the disruption and the remaining $11 \%$ of the warnings were issued $25 \mathrm{~ms}$ to $50 \mathrm{~ms}$ before the disruption. This comparison will be improved by increasing the number of discharges in the training set and applying both methods to the same discharges.

\section{References}

[1] Y.Martin and F.Bühlmann, Plasma Phys. Controlled Fusion, 40, 697, 1998.

[2] F.Ryter and H-mode database working group, Nucl. Fusion, 36, 1217, 1996.

[3] O.Kardaun, Classical Methods of Statistics with applications to fusion oriented plasma physics, Springer Verlag, to be published, 2004

[4] L.Giannone, A.C.C.Sips, O.Kardaun, F.Spreitler, W.Suttrop and the ASDEX Upgrade Team, Plasma Phys. Controlled Fusion, 46, 835, 2004.

[5] R.Gnanadeskin and J.Kettenring, Stat. Sci. Lett., 4, 34, 1989.

[6] F.Wagner et. al, Phys. Rev. Lett., 49, 1408, 1982.

[7] A.C.C.Sips et. al, Plasma Phys. Controlled Fusion,, 44, B69, 2002.

[8] G.Pautasso, C.Tichmann, S.Egorov et. al, Nucl. Fusion, 42, 100, 2002.

[9] B.Cannas et. al, P1-167, this conference. 

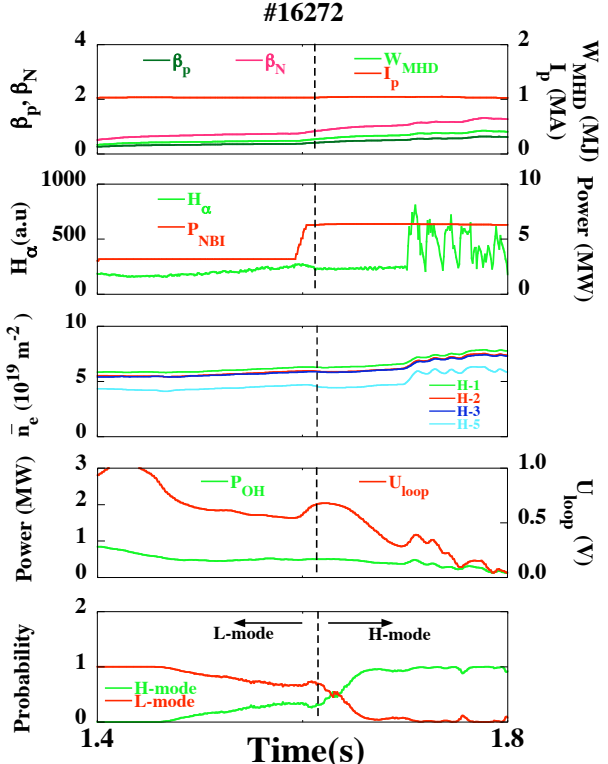

Figure 1: Overview of plasma parameters at the transition time to the H-mode. The regime identification probabilities for the $L$ mode (red) and H-mode (green) are shown in the bottom most plot.

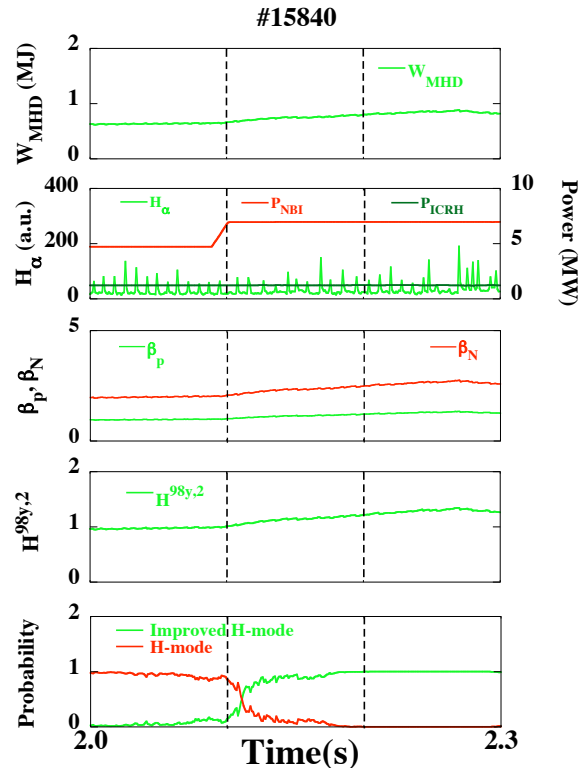

Figure 2: Overview of plasma parameters at the transition time to the improved H-mode. The regime identification probabilities for the ordinary H-mode (red) and improved H-mode (green) are shown in the bottom most plot. Between $2.1 \mathrm{~s}$ and $2.2 \mathrm{~s}$, the density and temperature profiles evolve as a consequence of the transition to the improved H-mode.

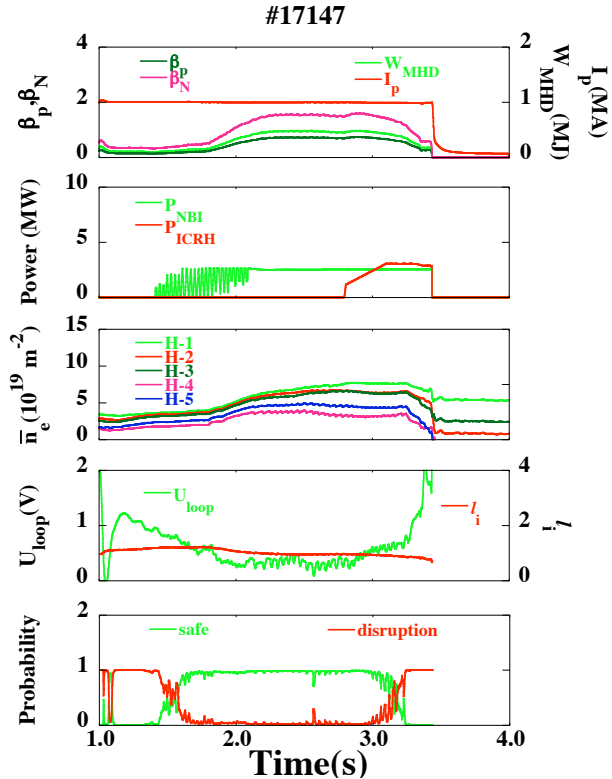

Figure 3: The time evolution of plasma parameters in a discharge with $B_{T}=2.0 \mathrm{~T}$ and $I_{p}=1.0 \mathrm{MA}$ that ends in a disruption. The regime identification probabilities for the safe (green) and disruption (red) regimes are shown in the bottom most plot.

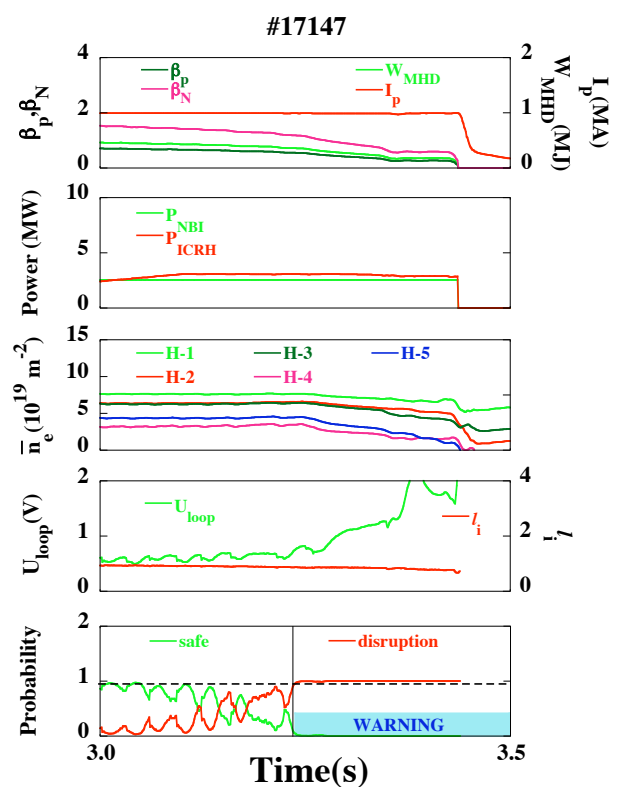

Figure 4: The disruption occurs at $3.44 \mathrm{~s}$ and the disruption warning is issued at $3.24 \mathrm{~s}$ when the probability reaches 0.93 . The regime identification probabilities on an expanded time scale for the safe (green) and disruption (red) regimes are shown in the bottom most plot. 